\title{
Particle Acceleration at the Pileup Collision of the Twin Shock
}

\author{
Xin Wang ${ }^{1,2,3,4,5}$ (D) , Joe Giacalone ${ }^{2}$, Yihua Yan $^{3}$, Mingde Ding ${ }^{4}$ (D), Chuan $\mathrm{Li}^{4}$ (D), Hong Lu ${ }^{6}$, and Hao Shan ${ }^{1,5}$ \\ ${ }^{1}$ Xinjiang Astronomical Observatory, Chinese Academy of Sciences, Urumqi 830011, People's Republic of China; wangxin@xao.ac.cn \\ ${ }^{2}$ Lunar and Planetary Laboratory, University of Arizona, Tucson, AZ 85721, USA \\ ${ }^{3}$ CAS Key Laboratory of Solar Activity, National Astronomical Observatories, Beijing 100012, People's Republic of China \\ ${ }^{4}$ Key Laboratory of Modern Astronomy and Astrophysics (Nanjing University), Ministry of Education, Nanjing 210093, People's Republic of China \\ ${ }_{5}^{5}$ Key Laboratory of Radio Astronomy, Chinese Academy of Sciences, Nanjing 210008, People's Republic of China \\ ${ }^{6}$ Key Laboratory of Particle Astrophysics, Institute of High Energy Physics, Chinese Academy of Sciences, Beijing 100049, People's Republic of China \\ Received 2018 July 18; revised 2019 September 2; accepted 2019 September 18; published 2019 October 31
}

\begin{abstract}
Ground-level enhancement (GLE) events are often associated with large gradual solar events such as fast coronal mass ejections (CMEs), but not all fast CMEs lead to GLE events. Is there a type of coordinated CME that could produce GLEs with larger intensity and higher energies than those in the normal fast isolated CMEs? Here we propose a twin-shock scenario driven by the twin CME coordinately, in which the posterior shock catches up with the preceding shock and has a pileup collision. In the present study, we chose the first GLE event of the solar cycle 24 occurring on 2012 May 17 as an example to investigate the probable association with the twin-shock scenario. We use a dynamic Monte Carlo method to examine the energy spectrum with relevance to the GLE event. In the twin-shock scenario, the seed energetic particles produced by the normal preceding shock can be injected into the posterior shock for reacceleration efficiently. As a result, we obtain the detailed energy spectrum of the solar energetic particles (SEPs) with different behaviors at the related episodes of the twin-shock evolution. Therefore, we predict that the pileup collision of the twin shock would dominate a concave energy spectral slope in the 2012 May 17 SEP event.
\end{abstract}

Key words: acceleration of particles - shock waves - solar wind - Sun: coronal mass ejections (CMEs)

\section{Introduction}

Historically flares and filament eruptions were the only known major transient features in the solar atmosphere. High energetic particles from the Sun were first observed (Forbush 1946) as sudden increases in intensity in ground-level ion chambers during the large solar events of 1942 February and March. Solar energetic particle (SEP) events were considered as a consequence of flares until the discovery of the coronal mass ejections (CMEs) in the 1970s (Kahler et al. 1978, 1984, 1987). Since the CMEs have been verified, a bimodal framework for the interpretation of SEP events has emerged. SEP events have been classified into the impulsive and gradual solar events (e.g., flares and CMEs; Cane et al. 1986; Reames 1995, 1999). Thereafter, there are a substantial amount of statistic studies and observational evidence to indicate that gradual SEP events can be associated with CMEs. For example, there is a linear correlation between the logarithmic of proton peak intensity and the logarithmic of speed of their associated CME (Kahler \& Vourlidas 2013). In the upstream region of a shock associated with the Bastille Day CME, the excitation of hydromagnetic waves with power spectral density levels well above the levels in the ambient solar wind was observed by Bamert et al. (2004) with the magnetometer on board the Advanced Composition Explorer (Mason et al. 1999). As another empirical association, the peak flux of the SEP is correlated more with the CME speed than with the X-ray flare peak flux (Gopalswamy et al. 2002).

However, one key question regarding SEPs within a space weather framework is: what type of events can give rise to extreme SEPs leading to a ground-level enhancement (GLE) event (Klein \& Dalla 2017)? In this paper we provide modeling efforts related to SEPs, with the aim of addressing this question.
Impulsive SEP events are short (less than 1 day), low intensity, and numerous (with an average number of about $1000 \mathrm{yr}^{-1}$ ). Gradual SEP events are long (several days) at energies of a few $\mathrm{MeV}$ nucleon $^{-1}$, rather rare for dozens per year, and orders of magnitude more intense in protons than impulsive SEP events. The gradual events, characterized by the largest proton fluxes and therefore of most relevance to space weather, are ascribed to acceleration by CME-driven shocks as they propagate through the heliosphere. In gradual events, protons can be accelerated into the $\mathrm{GeV}$ range, which may lead to neutron monitors (NMs) detecting events at Earth's surface. These GLEs are the most extreme of solar events with their special interests to the heliophysics community (Nitta et al. 2012; Asvestari et al. 2017). As a result of advances in instrumentation and an abundance of events to observe, we are understanding the energetic solar events and specifically GLEs that increased dramatically during solar cycle 23 (Gopalswamy et al. 2012). There were 16 GLEs in total in solar cycle 23. Comparatively, there is much quiet in solar cycle 24 , with only a few unambiguous GLE events. GLE events are one type of large gradual SEP event, which could be observed by ionization chambers and NMs on the ground. Protons and ions in these events are accelerated to very high energies, with intensities often 10-100 times larger than normal gradual SEP events. Since the observed particle temporal intensity profiles of GLEs are similar to those of normal gradual SEP events, we suggest that the underlying acceleration of GLE events should be the same as that operating in normal gradual SEP eventsthe diffusive shock acceleration (DSA; Ellison et al. 1990; Bykov et al. 2014). The standard DSA theory was born in the 1970s (Axford et al. 1977; Krymsky 1977; Bell 1978; Blandford \& Ostriker 1978), which requires a turbulent coronal medium to exist ahead of the CME-driven shock. It is well known that such conditions can be created ahead of the shock 
by the accelerated particles themselves through the instability of Alfvén waves resonant with the streaming accelerated particles (Bell 1978). Quantitative models employing this scenario have so far relied on quasi-stationary descriptions of the coupled particle acceleration and wave generation process (Zank et al. 2000; Li et al. 2003; Rice et al. 2003; Lee 2005). Its application in the interplanetary shocks such as CME-driven shocks has been noted by various research groups. Lee $(1983,2005)$ solved the coupled particle transport and wave action equations; this approach provided a self-consistent formalism for evaluating Alfvén wave amplification and particle energization at a quasi-parallel shock. Using the steady-state solution of the coupled system, Gordon et al. (1999) examined particle acceleration at Earth's bow shock. Later this formalism was extended by Lee (2005), who considered explicitly a two-stream approximation of the energetic particles. $\mathrm{Ng}$ et al. (2003) used the same equations as Lee (1983) but solved the time-dependent wave transport equation. Such an approach allows one to obtain the wave acceleration and energetic particle spectrum in a timedependent manner. On the other hand, Zank et al. (2000) and Li et al. (2003, 2005) assumed an instantaneous steady-state solution of the DSA and included the time consideration by balancing the shock dynamic time with the acceleration time. Using this approach, Li et al. (2009) examined the spectral break behavior in large SEP events and argued that the spectral breaks that are often observed must be $Q / A$ dependent and the dependence is due to the shock geometry.

For investigating why GLEs have larger intensity and higher energies than the normal gradual SEPs, Li et al. (2012) proposed a twin-CME scenario for GLE events and large SEP events upon solar cycle 23. In this scenario, two CMEs go off closely in time from the same active region (AR). The preceding CME drives a shock that generates a very turbulent region downstream and produces the seed of the normal energetic particles. As the posterior CME plunges into the strong turbulent region downstream of the preceding shock, it will accelerate them to very high energies (Gopalswamy et al. 2013; Ding et al. 2014). Depending on whether there is reconnection between the magnetic field turbulence that occurred on the posterior CME-driven shock and that produced by the preceding $\mathrm{CME}$, the pre-accelerated ions inside the preceding CME's driver can be processed by the posterior CME, leading to an enhancement of ions that are compositionally interplanetary CME-like ( $\mathrm{Li} \&$ Zank 2005). In addition, Gou et al. (2017) analyzed a model on the impulsive flare on 2013 May 13. They deduced that two-step magnetic reconnections between double consecutive flare eruptions play major roles in the production of the impulsive SEP event (for convenience, here called the twin-flare scenario). In fact, they have found the RHESSI (Lin et al. 2002) observations shown a strong burst of hard X-ray and $\gamma$-ray emissions with hard electron spectra, exhibiting a soft-hard-harder behavior.

Our twin-shock scenario is based on the previous twin-CME scenario proposed by Li et al. (2012). The twin-shock scenario, focusing on the presence of two CME-driven shocks, resembles that of sympathetic eruptions. Sympathetic eruptions are multiple eruptions within a relatively short period of time, which may occur across different source regions, or in a single complex source region (Ding et al. 2006, 2013; Zhukov \& Veselovsky 2007; Liu et al. 2009; Chen et al. 2011; Shen et al. 2013). If two coordinated eruptions from the same AR occur close in time and both drive shocks, then one finds both an excess of seed population and an enhanced turbulence level in front of the main shock, leading to very efficient particle acceleration. Because the twin-shock scenario suggests that efficient acceleration occurs at the shock driven by the posterior $\mathrm{CME}$, it implies that a single CME-driven shock may not lead to very large SEP events. Wang et al. (2016) examined an isolated CME-driven shock, which can just accelerate the proton to the maximum particle energies of SEP event up to $\sim 5 \mathrm{MeV}$. Ding et al. (2013) tested the twin-CME scenario against all large SEP events and western CMEs with speed $>900 \mathrm{~km} \mathrm{~s}^{-1}$ in solar cycle 23 . They found that most of the large SEP events agree with the twin-CME scenario, and many fast single CMEs do not lead to a large SEP event. Wang et al. (2017) also investigated the converging-shock scenario (e.g., the CME-driven shock with a head-on collision with Earth's bow shock), which can produce the maximum particle energies up to $\sim 20 \mathrm{MeV}$ greater than $E_{\max }$ in a single CME-driven shock, but forms a broken energy spectral slope at the energy range of $\sim 5.5 \mathrm{MeV}$. Here, we propose a twin-shock scenario with large differences from the converging-shock scenario. The twin-shock scenario focuses on the pileup effect on the SEP's production when the posterior shock catches up with the preceding shock. We suggest that the seed particles inside the preceding shock can be injected into the posterior shock for reacceleration efficiently.

For the 2012 May 17 GLE event, we compare the observations from multiple spacecraft at different heliospheric locations. Within the magnetosphere, proton energy spectra were measured by the Payload for Antimatter Exploration and Light-nuclei Astrophysics instrument (Picozza et al. 2007) as reported by Adriani et al. (2015), indicating that protons with energies of up to $1 \mathrm{GeV}$ and helium of up to $100 \mathrm{MeV}$ nucleon ${ }^{-1}$ were accelerated and transported to the vicinity of Earth. The $\mathrm{GeV}$ proton detection was also verified later by Kühl et al. (2015) using an inversion technique exploring the response functions of the Electron Proton Helium Instrument (Müler-Mellin et al. 1995) aboard the Solar and Heliospheric Observatory (SOHO) spacecraft. During this event, the STEREO Ahead (STA) and STEREO Behind (STB, Howard et al. 2008) spacecraft were leading and trailing Earth by $114^{\circ} .8$ and $117^{\circ} .6$, respectively, both at a heliocentric distance of $\sim 1$ au. Lario et al. (2013) studied the 15-40 MeV and $25-53 \mathrm{MeV}$ proton channels of this event using GOES and the high-energy telescope on STB. For the $15-40 \mathrm{MeV}$ channel, they obtained an enhancement rate (peak intensity/pre-event intensity) of $2.64 \times 10^{3}$ at GOES and only 35.0 at STB. For the $25-53 \mathrm{MeV}$ channel, they obtained an enhancement rate of $1.94 \times 10^{4}$ at GOES and only 13.4 at STB. Heber et al. (2013) included $S T A$ and $S T B$ proton time profiles for a single energy range in a figure, displaying the longitudinal extent of the event. The event was also observed by the MESSENGER spacecraft orbiting around Mercury, which, at the time of the event, was at a heliocentric distance of 0.34 au (Lawrence et al. 2016). Battarbee et al. (2018) investigated the time series of proton measurements from MESSENGER using its neutron spectrometry. Beyond $1 \mathrm{au}$, this event was also observed by the Radiation Assessment Detector (RAD; Hassler et al. 2012) on board the Mars Science Laboratory on its way to Mars (Zeitlin et al. 2013). The RAD detector did not measure original proton intensities in space, but rather a mix of primary and secondary particles due to primaries experiencing nuclear 
The Schematic Diagram of the Simulation Box in Twin-Shock Model

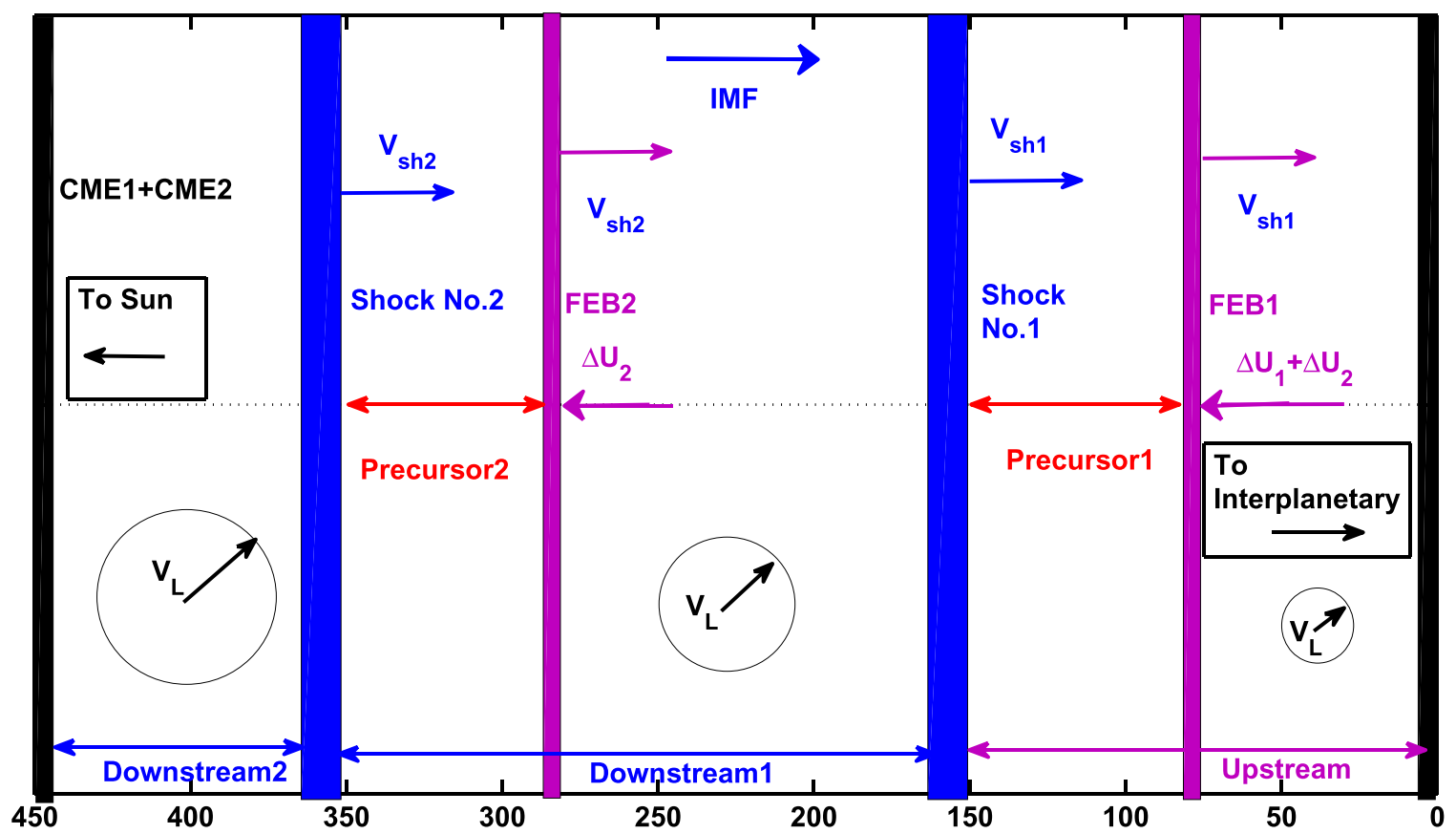

Figure 1. Schematic diagram of the twin-shock simulation box. The left reflective wall represents the twin CME producing shock No. 1 and shock No. 2 sympathetically (indicated by two blue vertical bars). Both shocks propagate from the left boundary into the simulation box separately. When shock No. 1 propagates into the center of the simulation box, shock No. 2 begins to catch up with shock No. 1 from the left boundary of the simulation box. Then the twin shock is evolving with a pileup interaction in the simulation box.

and electromagnetic interactions as they traverse through the inhomogeneous flight-time shielding of the spacecraft. Utilizing lower particle energies for release time analysis, $\mathrm{Li}$ et al. (2013) compared Wind/3DP (Bougeret et al. 1995) and GOES 13 particle fluxes with NM and solar disk observations, concluding that electrons at this event appear to be flareaccelerated, with proton acceleration happening mainly at the CME-driven shock. The presence of accelerated high-energy ions at the Sun is evidenced by $>100 \mathrm{MeV}$ gamma-ray emission (Ajello et al. 2014). Here, we attempt to further investigate whether the twin-shock scenario plays a more important role than an isolated CME-driven shock associated with the related SEP event.

This paper is organized as follows: A description of the model is presented in Section 2. The series of the simulated results of the twin-shock scenario are given in Section 3. Some discussions are given in Section 4. In Section 5, we present the summaries and conclusions.

\section{Model}

This model describes the twin-shock interactions of the 2012 May 17 SEP event at the interplanetary space within $7.5 R_{\odot}$, which would trigger the related GLE event at Earth's surface. Since the solar AR is located at W88 from Earth, the event was a backside event for STB. The propagation directions of the two CMEs are also shown in coronagraph observations made by $\mathrm{SOHO} / \mathrm{LASCO}$. While the two CMEs were clearly seen from $S T B$ and $S O H O$, they overlapped and appeared to be propagating together and were hardly distinguishable from the STA observations. Shen et al. (2013) use the Graduated Cylindrical Shell model to give the height $h$ of the two CMEs at different times and the parameters of the longitude $\Phi$ and the latitude $\Theta$. The differences of $\Delta \Theta$ and $\Delta \Phi$ between the two
CMEs are $11^{\circ}$ and $31^{\circ}$, respectively. This means that there are two distinct CMEs with a little difference of the propagation direction in this event. According to the calculated list of the height of the two CMEs at different times, the height $h_{1}=4.5 R_{\odot}$ for $\mathrm{CME} 1$ and the height $h_{2}=4.82 R_{\odot}$ for CME2 at the time of 02:00 UT are given. The difference of the heights between the two CMEs is $0.32 R_{\odot}$. They also used the relationship between the height of CMEs and the propagation time (Thernisien et al. 2009) to obtain the speeds of two CMEs, which are $1258 \pm 352 \mathrm{~km} \mathrm{~s}^{-1}$ and $1539 \pm$ $352 \mathrm{~km} \mathrm{~s}^{-1}$ for CME1 and CME2, respectively. Apparently the speed of the CME2 is greater than the speed of the CME1.

Figure 1 shows a schematic diagram of the twin-shock model. The left reflective wall represents two CMEs and produces the shocks at different times. Shocks No. 1 and No. 2 propagate from the left boundary of the simulation box to the right boundary. After shock No. 1 propagates into the center of the simulation box, shock No. 2 begins to pursue shock No. 1 with a relative speed $\Delta U_{2}$ from the left reflective wall. The two blue vertical bars at the simulation box indicate the shock No. 1 and shock No. 2 fronts, respectively. Initially, the upstream bulk flow speed of shock No. 1 is $\Delta U_{1}$ and the downstream bulk flow speed of shock No. 1 is zero at the rest shock No. 1 reference frame. When shock No. 2 enters into the simulation box, then two shocks begin to have an interaction with each other with a relative speed of $\Delta U_{2}$. Once the rest shock No. 1 reference frame transfers to the rest shock No. 2 reference frame, both of the upstream and downstream bulk speeds of shock No. 1 should be added an increment of the bulk speed $\Delta U_{2}$. Then, they become the $\Delta U_{1}+\Delta U_{2}$ and $\Delta U_{2}$ in the new rest shock No. 2 reference frame. The upstream and downstream bulk speeds of shock No. 2 also correspondingly become the $\Delta U_{2}$ and zero. In the twin-shock scenario, because there exists a relative bulk speed $\Delta U_{2}$ between the two 
sympathetic shocks, the downstream bulk flow of shock No. 1 is always compressed by shock No. 2 until shock No. 2 catches up with and exceeds shock No. 1. So the heated and accelerated ions inside the downstream of shock No. 1 can be seen as the seed of the energetic particles for reaccelerating by the Fermi mechanism at shock No. 2. We apply a dynamic Monte Carlo technique to simulate this twin-shock scenario in detail for investigating the integral energy spectral characters.

We consider double quasi-parallel shocks related to the 2012 May 17 SEP event where the supersonic flows move from the Sun to the interplanetary space along the $x$-axis direction, with the preceding shock propagating from the Sun along the $x$-axis to the backside of the extended line of the $S T B$ to the Sun. The posterior shock propagates along the largely same direction but with a little deviation of latitude angle $\Delta \Theta=-11^{\circ}$. The coronagraph perspectives of STEREO/COR1 and SOHO/C2 provide the observations of the collision of the twin shock. In order to investigate the enhancement intensity of the 2012 May 17 GLE event, we use a particle simulation method to reconstruct the energy spectrum of the related SEP event. The first GLE event on the solar cycle 24 provided an excellent chance for us to examine the ability of the twin-shock scenario for enhancing the intensity of the related SEP event, and we also present a comparison of the simulated results to the multiple spacecraft observations. Here, we employ a nonlinear dynamic Monte Carlo method to simulate the twin-shock scenario containing the back-reactions of the accelerated particles on the upstream subshock in front of the two shocks, respectively. In this model, shock No. 1 initially produces the heating and accelerated ions as the seed energetic particles for further acceleration when they penetrate into shock No. 2. Shock No. 2 moves forward to shock No. 1 with a relative bulk speed $\Delta U_{2}$ and a little deviation of the latitude angle $\Delta \Theta$. According to the data from the multiple spacecraft, we take the relative bulk speed of shock No. 1 to be $\Delta U_{1}=800 \mathrm{~km} \mathrm{~s}^{-1}$ between its upstream and downstream bulk flows, the relative bulk speed of shock No. 2 to be $\Delta U_{2}=400 \mathrm{~km} \mathrm{~s}^{-1}$ between its upstream and downstream bulk flows, and the background solar wind bulk speed to be $U_{0}=400 \mathrm{~km} \mathrm{~s}^{-1}$. Here, the downstream of shock No. 1 can be seen as the upstream of shock No. 2.

In this Monte Carlo method, we apply an initial number density of particles $n_{0}$ in the upstream bulk flow of shock No. 1 , which obeys a Maxwellian distribution with a thermal speed $v_{L 0}$. Shock No. 1 remains the relative bulk speed $\Delta U_{1}$ between the upstream and downstream bulk flow until shock No. 2 appears at the left boundary of the simulation box. The total simulation time is $T_{\text {tot. }}$ At half of the $T_{\text {tot }}$, we start shock No. 2 to pursue shock No. 1 with a relative speed $\Delta U_{2}$ from the left boundary of the box. During the next half of the total simulation time, the double shocks undergo a pileup collision, leading to the magnetic field amplification (MFA) and the energetic ions' reacceleration efficiently. As the second shock enters into the simulation box, the old shock No. 1 reference frame will be replaced by the new shock No. 2 reference frame. At the new rest shock No. 2 reference frame, both the upstream and downstream bulk speeds of the old shock No. 1 accordingly obtain an increment of the bulk speed $\Delta U_{2}$, respectively. The upstream and downstream bulk speeds of shock No. 2 become $\Delta U_{2}$ and zero, respectively. In any shock reference frame, the background solar wind bulk speed holds onto its value of $U_{0}$. Initially, we suggest shock No. 1 as a single CME-driven shock that produces a normal power-law energy spectrum at the first half of the total simulation time. Then, at the beginning of the next half of the total simulation time, shock No. 2 is starting to catch up with shock No. 1 and dramatically modifying the existing normal power-law energy spectrum inside the downstream region of shock No. 1. The additional modifications provided by shock No. 2 would change the old energy spectrum produced by shock No. 1. It is worth mentioning that the twin-shock scenario would differ from another type of converging-shock scenario (Wang et al. 2017), in which the CME-driven shock would be frustrated by the bow shock of the planet, resulting in a broken energy spectrum. But in this twin-shock scenario, since one CMEdriven shock catches up with the preceding CME-driven shock and they have a pileup collision when they propagate in the same direction, the positive effect of the twin shock on the energetic particles would enhance the existing energy spectrum. We predict that there probably appears a concave energy spectrum at a certain energy range on the related SEP event.

The great merit of the Monte Carlo method is applying the prescribed scattering law in the simulation. It is not necessary to differ the suprathermal particles from the thermal background bulk flow a priori. If the prescribed scattering law (e.g., isotropic and elastic scattering) is assumed, then the entire scattering processes can be decided for particle acceleration and the shock formation self-consistently. Using the Monte Carlo method for simulating shock formation and particle acceleration, it will not calculate the specific complicated magnetic field. This method considers that the initial thermal particles with the Maxwellian distribution embedded in the background solar wind will become the seed suprathermal particles if they cross the shock and are heated by the downstream turbulent magnetic field. Once these seed suprathermal particles possess the mean free path $\lambda_{\text {mfp }}$ beyond the shock transition width, then the particle can swim successfully upstream across the subshock through the post-shock cyclotron-instability-generated magnetohydrodynamic waves. Simultaneously, the injected particles via the back pressure smooth the existing shock transition to create a shock precursor (i.e., subshock), and further the particles would have multiple chances of shock crossing for achieving energetic particles. If there is no free escape boundary (FEB) in the far away upstream region to cut off the largest energetic particles, then the particles will keep on accelerating by using very long distance for returning particles back to the shock front. In the limited computational simulation consumption, we usually set a proper size of the FEB for satisfying the expectant energetic particle that can be produced. So in the Monte Carlo method, the injection rate is solely decided by the scattering law; it is not controlled as a free parameter (Caprioli et al. 2010). If there is no FEB, the largest energetic particles would not escape and achieve the unexpected largest energetic particle. We take the isotropic and elastic scattering law in this twin-shock model; the particle injection and escape will be treated in a natural and self-consistent manner.

The scattering process is usually modeled by some dependencies of the mean free path $\lambda_{\mathrm{mfp}}$ of the particles on momentum $p$ in most particle simulation cases. Thus, in order for the acceleration to be efficient, a large number of shock 
crossings back and forth on the shock front are required. Shock particle acceleration therefore depends on the scattering process, which resembles a stochastic process, depending on the presence of scattering centers upstream and downstream and on the random changes in the scattering angles. It assumes that the scattering is elastic and isotropic for conserving energy. This mechanism always works until the gyroradius of the accelerated particle exceeds the size of the system or the energy of the particle becomes so large that the back-scattering is ineffective. In order to experience the first scattering, the particle must possess a gyroradius much larger than the entire width of the shock transition region (Treumann 2009). Only when this condition is satisfied will the shock behave like a slightly thin discontinuity separating two regions of very different bulk speeds. A particle crossing back and forth over such a shock can become aware of the bulk difference in speed and gain energy additions. In fact, for entering into the Fermi acceleration mechanism a particle must be preheated until its gyroradius becomes larger than the width of the shock transition region.

The Monte Carlo method applies a scattering law for particle diffusive processes on shocked plasmas. We assume that the particles scatter elastically off the background scattering centers with their scattering angles obeying an isotropic distribution in their local frame. In this scattering scenario, the assumption of elastic scattering requires that scattering centers are frozen into the background fluid. Simultaneously, the assumption of a constant collision time for all particles requires that the particle's mean free path is proportional to its local thermal velocity in the local frame (Knerr et al. 1996; Wang \& Yan 2011, 2012; Wang et al. 2013; Ellsion et al. 2016). For simplicity, we take its formula as

$$
\lambda_{\mathrm{mfp}}=v_{L} \cdot \tau
$$

For pitch-angle scattering, the particle momentum vector undergoes a pitch-angle deflection $\delta \theta$ based on the dispersion of the turbulent magnetic field $\delta B / B$. Individual particles will undergo such small deflections in the scattering processes. When an individual particle's accumulation of such small pitch-angle deflections $\sum=\delta \theta_{1}+\delta \theta_{1}+\ldots+\delta \theta_{n}$ achieves $\Delta \theta=90^{\circ}$, then it can be seen to fulfill a collision process. The time consumption for completing this collision process can be defined as the collision time $\tau$. But in this entire scattering process, we need to take an average collision time (i.e., $\tau=$ constant) according to the local turbulent background magnetic field. So the constant collision time has the relationship from Equation (1) as formula $\tau=\lambda_{\mathrm{mfp}} / v_{L}$, where $v_{L}$ is the particle's local velocity in its local frame. It was shown in Ellsion et al. (2013) that the maximum angle deflection for individual particles in an interaction satisfies

$$
\delta \theta_{\max }=\sqrt{6 \delta t / \tau}
$$

where $\delta t$ is the time in the local frame between the pitchangle scatters. This scattering process is simulated with two random numbers, $\varphi$ and $\delta \theta$. At each time step $\delta t$, the azimuthal angle $\varphi$ is chosen randomly between 0 and $2 \pi$, and the pitch angle, $\delta \theta$, is chosen from a uniform distribution of $\cos \delta \theta$ between 1 and $\cos \delta \theta_{\max }$ (Ellsion et al. 2013). The average number of scattering events occurring in the time step $\delta t$ depends on the average collision time $\tau$, and the scattering rate $R_{s}$ is presented by

$$
R_{s}=\delta t / \tau
$$

where $R_{s}$ is the probability of the scattering events occurring in an increment of time step. The candidates with their local velocities and scattering angles scatter off the grid-based scattering centers. These individual particles do not change their routes until they are selected to scatter once again. For the individual proton, the grid-based scattering center can be seen as a sum of individual momenta. So these scattering processes can be taken as the elastic collisions. In an increment of time step, once all of the candidates fulfill these elastic collisions, the momentum of the grid-based scattering center is changed. The total simulation temporally evolves forward by repeating the time step sequences. In order to calculate the scattering processes accurately and produce an exponential mean free path distribution, the time step should be much less than the collision time (i.e., $\delta t<\tau$ ). In these scattering processes, the particles in the upstream region transfer their kinetic energy into their random thermal energy in the downstream region. Then, the minority of random thermal particles can be injected from the downstream region into the upstream region by multiple scattering cycles on shock fronts to obtain more energy gains and become the energetic particles.

Specifically, the twin-shock Monte Carlo code is set up much like a typical hybrid code (Ellison et al. 1993): particles are moved under the influence of existing fields, moments are collected (i.e., the mass and current density are found via a grid-based summation over all particles), and new fields are calculated. The major difference is that there is no field calculation. This step is replaced by a simple statistical decision as to whether or not each particle scatters. In summary, the dynamical method consists of three main steps (Knerr et al. 1996):

(i) Make the particles move. Movement equations of the particles obey their velocities in the $\hat{x}$-direction

$$
X_{k}^{t}=X_{k}^{t-1}+\left(v_{x}\right)_{k}^{t} d t, t \in\left[1, t_{\mathrm{tot}}\right], k \in\left[1, k_{\max }\right],
$$

where

$$
\begin{gathered}
\left(v_{x}\right)_{k}=\left(v_{L x}\right)_{k}+U_{k}, \\
U_{k}=\frac{1}{n_{k}} \sum_{i=1}^{n_{k}}\left(v_{x}\right)_{i},
\end{gathered}
$$

where $t_{\text {tot }}$ represents the number of the total time steps, which is equal to $T_{\text {tot }} / \delta t=36,000$; the number of the total computational grids is equal to $k_{\max }=900$; the subscript $k$ represents the index of the computational grid; $U_{k}$ represents the bulk fluid speed of the computational grid $k$ along the $\hat{x}$-direction; and the value of $U_{k}$ will be obtained from step (ii). Since the simulated twin shocks are plane-parallel shocks, fluid quantities only vary in the $\hat{x}$-direction.

(ii) Do the collections of the masses, velocities, and moments of the particles based on computational grids. These statistical processes are fulfilled by the standard particle-in-cell (PIC) technique. Collecting the moments involves the usual summing 
Table 1

The Simulation Parameters

\begin{tabular}{|c|c|c|}
\hline Physical Parameters & Dimensionless Values & Scaled Values \\
\hline Relative upstream bulk speed 1 & $\Delta U_{1}=0.6$ & $800 \mathrm{~km} \mathrm{~s}^{-1}$ \\
\hline Relative upstream bulk speed 2 & $\Delta U_{2}=0.3$ & $400 \mathrm{~km} \mathrm{~s}^{-1}$ \\
\hline Solar wind background bulk speed & $U_{0}=0.3$ & $400 \mathrm{~km} \mathrm{~s}^{-1}$ \\
\hline Initial thermal velocity & $v_{L 0}=0.02$ & $26.9 \mathrm{~km} \mathrm{~s}^{-1}$ \\
\hline Collision time & $\tau=0.833$ & $7.14 \mathrm{~s}$ \\
\hline Box size & $X_{\max }=450$ & $7.5 R_{\odot}$ \\
\hline FEB size & $\mathrm{FEB}_{1,2}=90$ & $1.5 R_{\odot}$ \\
\hline Total time & $T_{\mathrm{tot}}=2400$ & 343 minutes \\
\hline Time step size & $\delta t=1 / 15$ & $0.57 \mathrm{~s}$ \\
\hline Number of zones & $m_{x}=900$ & $\cdots$ \\
\hline Initial particles per cell & $n_{0}=300$ & $\ldots$ \\
\hline
\end{tabular}

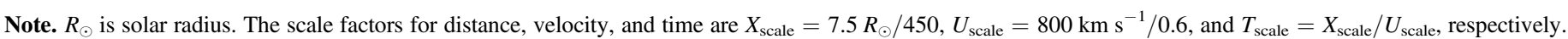
The dimensionless values and the scaled values can be transformed by the scaled factors.

of particle masses and velocities on a background computational grid

$$
\begin{aligned}
P_{k} & =\sum_{i=1}^{n_{k}} m_{p}\left(v_{x}\right)_{i}, k=1,2, \ldots k_{\max } \\
U_{k} & =\frac{1}{n_{k}} \sum_{i=1}^{n_{k}}\left(v_{x}\right)_{i}, k=1,2, \ldots k_{\max },
\end{aligned}
$$

where $n_{k}$ is the number density of particles in the $k$ grid, representing the mass of the computational grid, and it is calculated by the PIC method in the code. $P_{k}$ is the total momentum of the protons in the $k$ grid, $m_{p}$ represents the mass of a proton, and $U_{k}$ is the average bulk fluid grid speed. Under the assumption of the massless fluid of electrons (Leroy et al. 1982), we only consider the masses of the protons in the plasma in this step. These grid-based mass and momentum densities are then used to find the local bulk flow speed $U_{k}$ in Equation (8) in each grid zone (to be used as the velocity of the scattering centers for particles in the local grid). According to the value of $U_{k}$, the temporal shock structure could be determined dynamically. The particle's total velocity in box frame should be a formula like Equation (5). During the first shock formation, if the value of $U_{k}$ of the first downstream region becomes zero, we can decide the first boundary of the shock front dynamically. The value for the bulk speed of the first upstream region is $U_{k}=\Delta U_{1}$. As the second bulk flow enters into the simulation box, the value of $U_{k}$ for the second downstream will become zero, and the value of $U_{k}$ for the first downstream will become $\Delta U_{2}$. During the formation of both shocks, $U_{k}=\Delta U_{1}+\Delta U_{2}$ represents the first upstream region, $U_{k}=\Delta U_{2}$ represents the second upstream region, and $U_{k}=0$ represents the second downstream region in the new reference shock frame. As see from Figure 1, the two shocks are formed dynamically, and the two FEBs (e.g., FEB $_{1,2}=90$ ) in the front of their shocks dynamically move away from the left reflective wall with their evolution velocities $v_{\mathrm{sh} 1}$ and $v_{\mathrm{sh} 2}$, respectively.

(iii) Starting the pitch-angle scattering law in scattering processes. This step involves choosing a certain fraction of the particles in each grid zone to scatter. The average number of scattering events occurring in each time step depends on the choice of the scattering time $(\tau)$. This selected fraction in each time step is decided by Equation (3), where $R_{s}$ is the ratio of the scattering event occurring in the time step $(\delta t)$.

The selected particles scatter off the grid-based scattering centers with the given scattering angles $(\delta \theta, \delta \phi)$ according to the prescribed scattering angular distributions in each grid zone at any of the time steps, and with its local thermal velocity $v_{L x}$, $v_{L y}, v_{L z}$ along the $X-, Y$-, and $Z$-axis directions for its mean free path moving until this particle will be selected to scatter again. The mean free path is proportional to the local thermal velocities in the local frame as in Equation (1). In this step, we chose a certain fraction of the grid density particles for scattering off the background bulk scattering center. As far as a selected individual particle interacting with the collective background scattering center is concerned, we can see that the grid-based background scattering center moment is massive enough for an individual particle, and this scattering process would be taken as an elastic collision. After all selected particles fulfill their scattering processes completely in the local background cell center, their collective changes in moments not only will affect the local background cell's speed but also will affect other neighboring background cells' speed. Similarly, the local background center speed will be affected by the incoming particles from neighboring cells. So we can see that the dynamical changes in collective background bulk speed are contributed by a large amount of individual elastic scattering processes. In turn, the background bulk speed will affect the individual particle's velocity as seen in step (ii) reversely.

These three substeps make up one complete time step. Repeating this time step sequence evolves the system forward in time. The time step size is chosen to be less than the scattering time $(\delta t<\tau)$ to resolve the scattering accurately and produce an exponential mean free path distribution.

In this twin-shock simulation box, all the simulated parameters are listed in Table 1. According to the observations, we adjust the observed parameters for applying the appropriate simulated parameters. We present the scaled values of the parameters as follows. The background solar wind bulk speed is $U_{0}=400 \mathrm{~km} \mathrm{~s}^{-1}$. The relative upstream bulk flow speed of shock No. 1 is $\Delta U_{1}=800 \mathrm{~km} \mathrm{~s}^{-1}$; this means that the speed of CME No. 1 is $U_{\text {cme } 1}=U_{0}+\Delta U_{1}=1200 \mathrm{~km} \mathrm{~s}^{-1}$ in the laboratory reference frame. The relative upstream bulk flow speed of shock No. 2 is $\Delta U_{2}=400 \mathrm{~km} \mathrm{~s}^{-1}$; this means that the speed of CME No. 2 is $U_{\mathrm{cme} 2}=U_{0}+\Delta U_{1}+$ $\Delta U_{2}=1600 \mathrm{~km} \mathrm{~s}^{-1}$ in the laboratory reference frame. The initial local thermal velocity is $v_{L 0}=26.9 \mathrm{~km} \mathrm{~s}^{-1}$ in the 
background solar wind. ${ }^{7}$ The collision time is $\tau=7.14 \mathrm{~s}^{8}{ }^{\text {The }}$ time step is set to be $\delta t=0.57 \mathrm{~s}$. The box size is chosen to be $X_{\max }=7.5 R_{\odot}$ for ensuring the twin-shock interaction within the box (where $R_{\odot}$ is the solar radius, about 109 times the Earth radius $R_{e}$ ). The total time of the simulation is chosen to be $T_{\text {tot }}=343$ minutes. For simulation, the total time needs to be long enough for producing large SEP events. Although the solar wind bulk speed is varying with the radial distance, CME No. 1 and CME No. 2 with their speeds at a level above $1000 \mathrm{~km} \mathrm{~s}^{-1}$ would take $\sim 40-50$ minutes to pass through the domain from 1 to $7.5 R_{\odot}$ and would produce the variations of the CMEs' speeds at a level of $\sim 100 \mathrm{~km} \mathrm{~s}^{-1}$. We thought that these variations of the CMEs' speed would not substantially affect our simulations, because we just consider the CMEdriven shocks' evolutions and the relative bulk speed between the upstream and downstream region, but not the CMEs themselves bulk flows. In our simulation system, we set the CMEs steady on the left boundary of the simulation box, and we describe the CME-driven shocks moving with their evolution velocities $V_{\mathrm{sh} 1}$ and $V_{\mathrm{sh} 2}$, which are estimated to be $\sim 100-200 \mathrm{~km} \mathrm{~s}^{-1}$ in the local reference frame. This means that although the speed of the CMEs changes temporally, the shocks driven by the CMEs still evolute in front of the CMEs with their velocities in the local reference frame without substantial influence.

The above scaled values of the parameters correspond to the following dimensionless parameters, respectively: upstream bulk speeds $\Delta U_{1}=0.6$ and $\Delta U_{2}=0.3$; initial local thermal velocity $v_{L 0}=0.02$; constant of the collision time $\tau=0.833$; total size of the box $X_{\max }=450$; free escape boundary distance $\mathrm{FEB}_{1,2}=90$; total simulation time $T_{\text {tot }}=2400$; time step $\delta t=1 / 15$. These dimensionless values can be scaled by the distance $(X)$, time $(T)$, and velocity $(U)$ scaling factors: $X_{\text {scale }}=7.5 R_{\odot} / 450, \quad U_{\text {scale }}=800 \mathrm{~km} \mathrm{~s}^{-1} / 0.6$, and $T_{\text {scale }}=$ $X_{\text {scale }} / U_{\text {scale }}$, respectively. In addition, we give the simulation box grids of $m_{x}=900$, and the initial density of particles in each grid is $n_{0}=300$. The total number of the particles in the simulation box at the end of the simulation archives to more than 1 million particles.

\section{Results}

\subsection{Shock Evolution}

Figure 2 shows a series of the velocity snapshots of the bulk flow with the simulation grids at the simulation times of $T=240,480,720, \ldots$, and 2400. The first five panels show the bulk flow of a single shock with the upstream bulk speed of shock No. 1 of $\Delta U_{1}=0.6$ during the first half of the total

\footnotetext{
7 Here, the initial local thermal velocity $v_{L 0}$ is the same as the one in the previous simulation (Wang et al. 2017), because there is the same initial background solar wind bulk speed $U_{0}=400 \mathrm{~km} \mathrm{~s}^{-1}$.

8 The chosen value of the collision time $\tau$ is different from the one in Wang et al. (2017). The present simulation describes the shocks at the solar surface in about a few $R_{\odot}$ range, but the previous simulation located Earth at about 1 au distance. The collision time $\tau$ is correlated to the pitch-angle deflection $\delta \theta$, which is proportional to the local turbulent interplanetary magnetic field (IMF) $\delta B$. At the solar surface, the turbulent IMF can be characterized by $\delta B / B \odot \sim 10^{-4}, B_{\odot} \sim 1 \mathrm{G}$, and $\delta B_{\odot} \sim 0.1 \mathrm{mG}$. At the 1 au distance, the turbulent IMF can be taken as $\delta B / B \sim 1, B_{\text {au }} \sim 1-10 \mathrm{mG}$, and $\left\langle\delta B_{\text {au }}\right\rangle$ $\sim 5.5 \mathrm{mG}$. Comparatively, to fulfill a complete collision at the solar surface, we need more accumulations of the small pitch-angle deflections $\delta \theta$ than there are in the Earth location. So, at the average level, the collision time at the solar surface needs $\sim 55$ times the collision time at Earth orbit (i.e., $\tau_{R_{\odot}} \sim 55 \tau_{1 \text { au }}$ ). The previous simulation had the Earth location with $\tau_{1 \text { au }}=0.13 \mathrm{~s}$, so we take $\tau_{R_{\odot}}=7.14 \mathrm{~s}$ in the present simulation.
}

simulation time; the downstream bulk speed of shock No. 1 is zero. The last five panels show the bulk flows of the double shocks since shock No. 2 enters into the simulation box during the last half of the total simulation time. When the simulation starts from time $T=1200$, the new rest shock reference frame is built on shock No. 2, the upstream bulk speed of shock No. 1 becomes $\Delta U_{1}+\Delta U_{2}=0.9$, and the downstream bulk speed of shock No. 1 becomes $\Delta U_{2}=0.3$. The upstream bulk speed of shock No. 2 is also $\Delta U_{2}=0.3$, but the downstream bulk speed of shock No. 2 becomes zero. Comparatively, the first five panels show a single-shock evolution, and the last five panels show a twin-shock evolution. From the view of the sixth panel $(T=1440)$, shock No. 1 is located in grid number $m \sim 550$ and shock No. 2 is located in the simulation grid number $m \sim 720$. The region between the simulation grid number $m \sim 550$ and the grid number $\sim 720$ can be seen as the upstream bulk flow of shock No. 2 or the downstream bulk flow of shock No. 1. The common region between shock No. 1 and shock No. 2 is compressed with the relative bulk speed $\Delta U_{2}$. With the time increasing, shock No. 2 compressing the downstream bulk flow of shock No. 1 with the relative velocity of $\Delta U_{2}$ is clearly shown from the seventh panel $(T=1680)$. Then, we can see that the common region disappears and the downstream bulk flow of shock No. 1 and the upstream bulk flow of shock No. 2 merge together gradually in the eighth panel $(T=1920)$. At the rest merged twin-shock reference frame, since shock No. 2 has caught up with and exceeded shock No. 1, the upstream bulk speed of the twin shock is $\Delta U_{1}+\Delta U_{2}=0.9$, and the downstream bulk speed of the twin shock becomes zero. For shock No. 2, its high efficient acceleration is not only for the local thermal particles but more for the energetic particles that have been accelerated by shock No. 1. As an aspect of the pileup effect of the twin-shock scenario, there seemingly are seldom studies up to now. In this dynamic Monte Carlo simulation model, we can present the shock formation and the particle acceleration information with its random velocity at any momentum and position. We guess that the initial twin shock undergoes a pileup collision and merges into a large single shock ultimately. But its pileup effect on the accelerated particles should differ from that a normal single shock can have. These acceleration processes could be described by the three-step accelerations. At the first step, shock No. 1 translates the kinetic energies of the upstream bulk flow into the particle's random energies by the multiple shockcrossing cycles and produces a normal power-law energy spectrum. At the second step, shock No. 2 translates kinetic energies of the bulk flow of the common region into the existing energetic particles with power-law energy spectrum accelerated by shock No. 1 for reaccelerations up to the higherenergy spectrum. At the last step, as shock No. 1 disappears completely, the merged twin shock as a large single shock still modifies the energy spectrum of the enhanced SEP event.

Figure 3 shows a group of bulk flow velocity profiles at the simulation time durations of $Q=1,2,3, \ldots$, and 10 . The red solid line in each panel represents the position of shock No. 1, respectively. The yellow solid line in each panel represents the position of shock No. 2, respectively. From the first panel to the fifth panel, we can see only a single shock evolving with the bulk flow velocity profiles in terms of the position and the time. The left blue area of the red line in each panel represents the downstream region of shock No. 1, and the right green area of the red line represents the upstream region of shock No. 1. At 

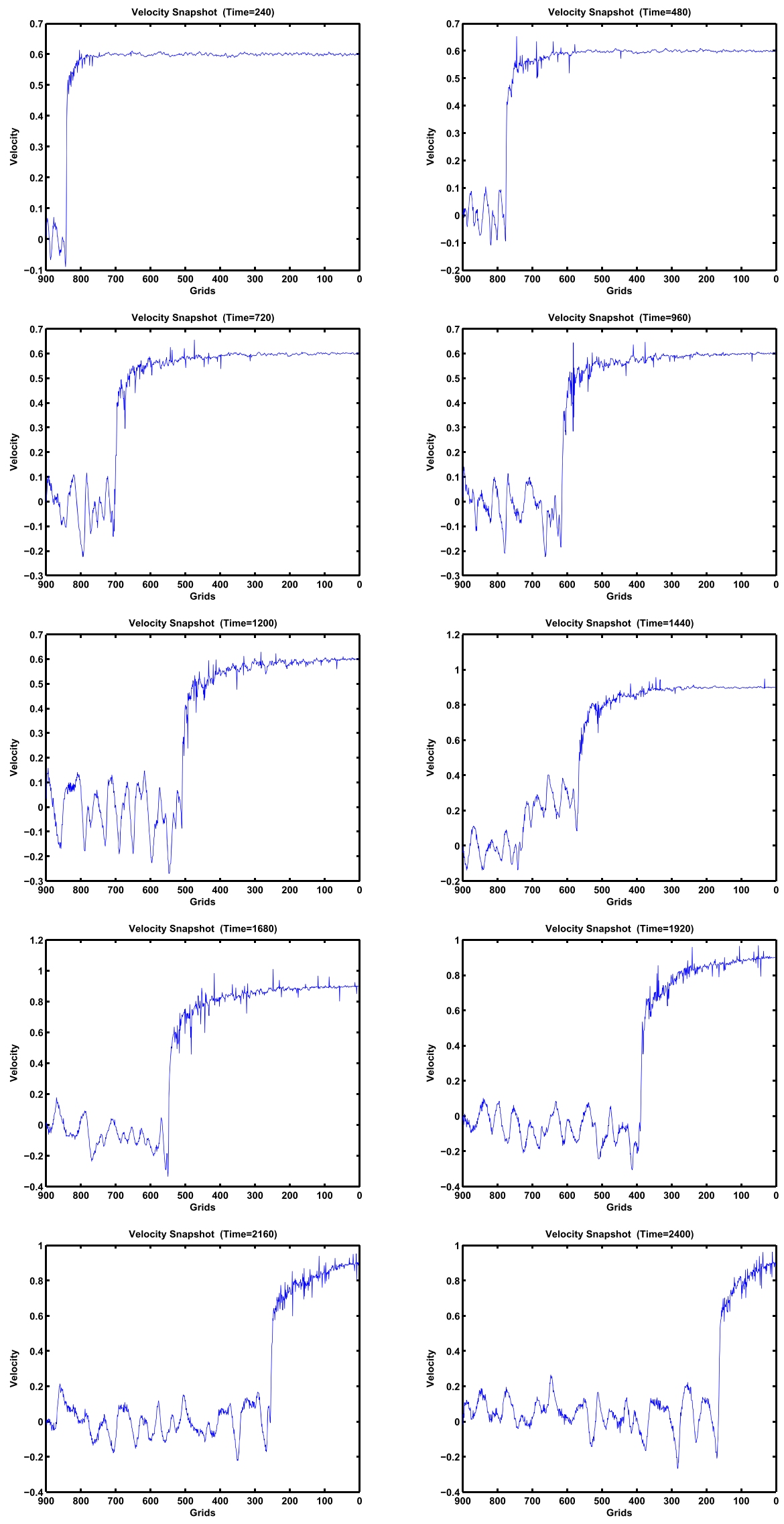

Figure 2. Velocity snapshots of the bulk flow with the grids at a series of simulation times $T=240,480,720, \ldots$, and 2400 . 

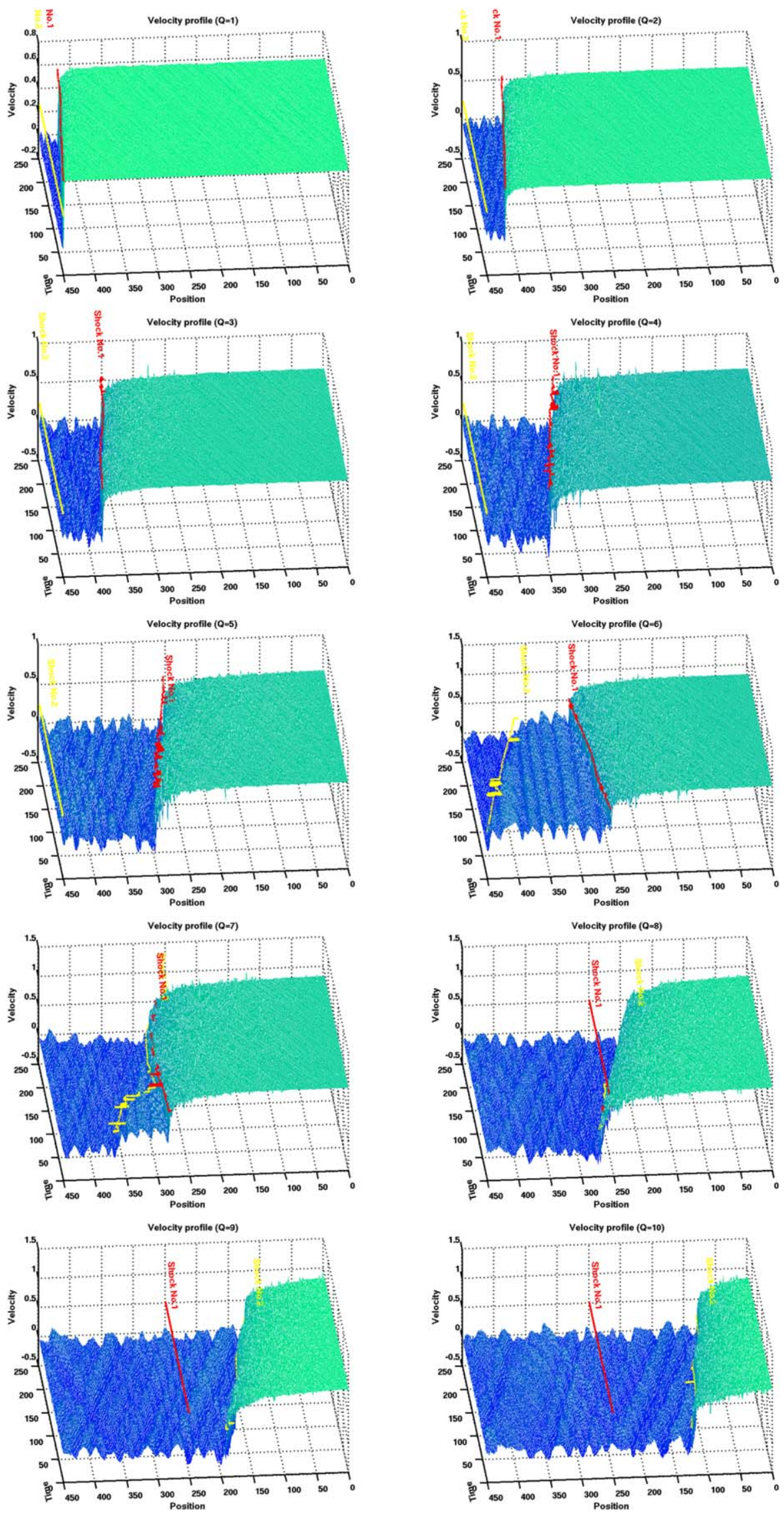

Figure 3. Velocity profiles of the bulk flow at the durations of simulation times $Q=1,2,3, \ldots$, and 10 . 
the rest shock No. 1 reference frame, the speed of the upstream bulk flow has an initial value of $\Delta U_{1}=0.6$, and the speed of the downstream bulk flow has a value of zero. The blue area of the downstream region gradually expands into a triangle shape during the first time duration $(Q=1)$. At the second time duration $(Q=2)$, the area of the downstream region evolutes from a triangle area into a trapezoid area. The area of the downstream region becomes wider and wider up to the fifth time duration $(Q=5)$. As viewing from the panels at the first five time durations $(Q=1 \sim 5)$, since shock No. 2 has not entered into the simulation box, the yellow line remains motionless at the left boundary of the simulation box. From the sixth time duration $(Q=6)$ on, the yellow line begins to move and form a new triangle region as the downstream bulk flow of shock No. 2. At the new rest shock No. 2 reference frame, the speed of the upstream bulk flow of shock No. 1 changes from $\Delta U_{1}=0.6$ to the new value of $\Delta U_{1}+\Delta U_{2}=0.9$. The speed of the downstream bulk flow of shock No. 1 changes from a value of zero to a value of $\Delta U_{2}=0.3$. For shock No. 2, the speed of the downstream bulk flow becomes zero, and the speed of the upstream bulk flow is $\Delta U_{2}=0.3$, with the same speed value for the downstream bulk flow of shock No. 1. At the sixth panel, we can see that two shock fronts move closely. At the left side of the yellow line, there is a new triangle downstream region of shock No. 2. The region between the yellow line and red line, shaped as an opposite trapezoid, represents the common region indicating that both shock No. 1 and shock No. 2 fronts move closer and closer gradually. From the sixth time duration $(Q=6)$ to the seventh time duration $(Q=7)$, the opposite trapezoid region between shock No. 1 and shock No. 2 fronts disappears approximately as the time increases. From both the sixth panel and the seventh panel, we can see that shock No. 2 has caught up with and exceeded shock No. 1 with the disappearance of the opposite trapezoid region. At the eighth panel, we can see that the red line remains motionless and the yellow line goes on moving forward to the right side direction. After the completion of the pileup interaction, the new merged twin shock still evolutes like a large single CME driven shock until the end of the simulation. Here, the speed of the upstream bulk flow of the merged twin shock shows a value of $\Delta U_{1}+\Delta U_{2}=0.9$, and the speed of the downstream bulk flow is zero. Here, we can calculate the shock evolution velocities for two shocks according to Figures 2 and 3 . The formulas $V_{\mathrm{sh} 1}=\left(X_{\max }-X_{\mathrm{sh} 1}\right) / T_{\text {half }}$, when $Q=5$, and $V_{\text {sh2 }}=\left(X_{\max }-X_{\text {sh2 }}\right) / T_{\text {tot }}$, when $Q=$ 10. We obtained the shock evolution velocities of $V_{\mathrm{sh} 1} \sim$ $194.39 \mathrm{~km} \mathrm{~s}^{-1}$ and $V_{\mathrm{sh} 2} \sim 208.40 \mathrm{~km} \mathrm{~s}^{-1}$ at their corresponding simulation times. For the particle acceleration, at the first five time durations, particles undergo the first-step acceleration by a single shock No. 1 and form a normal power-law energy spectrum. With the entrance of shock No. 2 and the formation of the opposite trapezoid region in the simulation box, shock No. 2 translates the kinetic energies of the common region into the existing power-law-distributed particles again for the second-step acceleration. With the common region and shock No. 1 disappearing gradually, the third-step acceleration by the merged twin shock has been carrying on, which is similar to a large single CME driven shock. But its pileup effect on the energy spectrum should be distinguished from the general behavior modified by a normal single shock.

\subsection{Particle Acceleration}

Figure 4 shows a series of panels for the particle acceleration processes at the sequences of the simulation time durations of $Q=1,2,3, \ldots$, and 10 . The blue curves in each panel trace a few particles' velocities increasing by multiple crossing cycles back and forth along the shock front with the position and time. The pink triangle shaded area represents shock front No. 1. The green triangle shaded area represents shock front No. 2. At the first five panels, the green triangle shaded area remains silent at the left boundary of the simulation box, and the pink triangle shaded area moves away from the initial position $(x=450)$ toward the inner part of the simulation box. With the time increasing, the pink triangle shaded area moves far away from the left boundary of the simulation box. We can see just a single shock front for particles' crossings back and forth in the first five panels. Since those particles obtain the first-step acceleration by crossing on the single shock No. 1, the particle energies just obey a normal power-law distribution according to DSA theory. As seen from the first five panels, the maximum particle velocity is promoted from the value of $V_{\max }=13.15$ at the first time duration $(Q=1)$ to the value of $V_{\max }=30.73$ at the fifth time duration $(Q=5)$. In the sixth panel at the sixth time duration $(Q=6)$, the green triangle shaded area begins to move into the inner part of the simulation box, and some of the particles also obtain energy gains by crossing on shock No. 2 . One of the particles obtains a maximum velocity value for $V_{\max }=34.6$, which is clearly injected from shock No. 1 to shock No. 2 according to its trajectory on the sixth panel. With inspection of the seventh panel, the green triangle shaded area (shock No. 2) moves closer and closer to the pink triangle shaded area (shock No. 1), quite a few particles are injected into shock No. 2 from shock No. 1, and more and more particles obtain the second-step acceleration by shock No. 2 . The maximum particle velocity abruptly achieves the value of $V_{\max }=42.30$. At the eighth time duration $(Q=8)$, the common region between two shocks has been compressed completely and shock No. 2 has already exceeded shock No. 1. Then, the pink triangle shaded area (shock No. 1) maintains the motionless state gradually, and the yellow triangle shaded area (shock No. 2) keeps on moving forward to the next time durations. The merged particles obtain the maximum velocity with the value for $V_{\max }=45.61$ at the eight time duration $(Q=8)$. From the ninth time duration $(Q=9)$ to the 10th time duration $(Q=10)$, the merged twin shock goes on propagating forward like a large single CME driven shock. The maximum particle velocity achieves the value of $V_{\max }=50.54$ at the end of the simulation. Although the merged twin shock is like a large single shock at the end, the realistic energetic particles have been accelerated separately by both shocks when shock No. 2 encounters shock No. 1. With the merging of the common region between shock No. 1 and shock No. 2 completely, the merged energetic particles go on undergoing the third-step acceleration by the merged twin shock, which is different from a normal single shock.

\subsection{Energy Spectra}

Figure 5 shows the simulated energy spectra at the end of the simulation with two types of representations for the particle fluxes. The blue curve represents the particle fluxes with the energy in normal representation. The red curve represents the particle fluxes multiplying by a factor of the square of the 

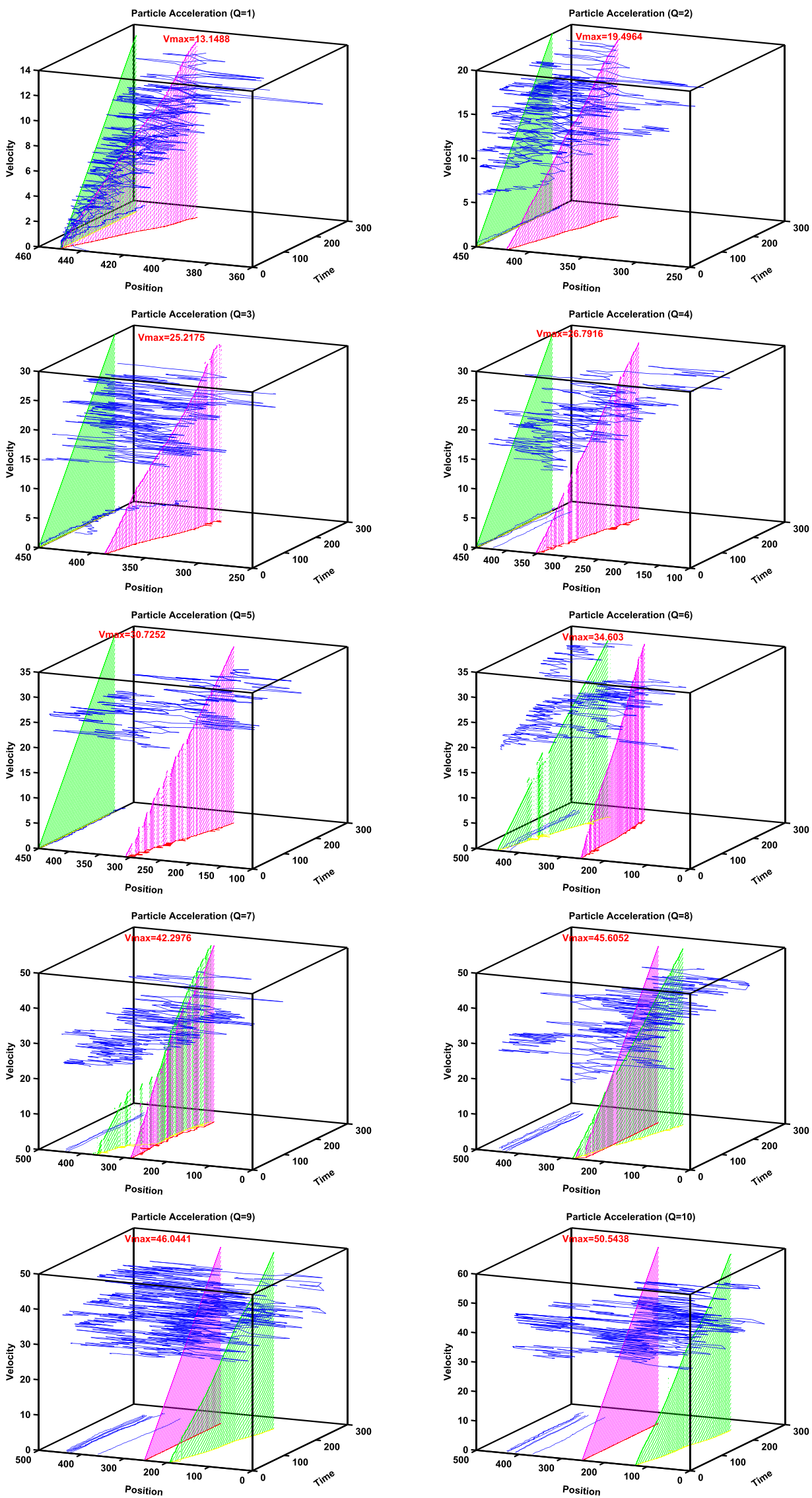

Figure 4. Particle accelerations in the simulation time durations $Q=1,2,3, \ldots$, and 10. The blue curves in each panel represent the particle trajectories. The pink and green triangular shaded areas represent shock No. 1 and shock No. 2, respectively. 


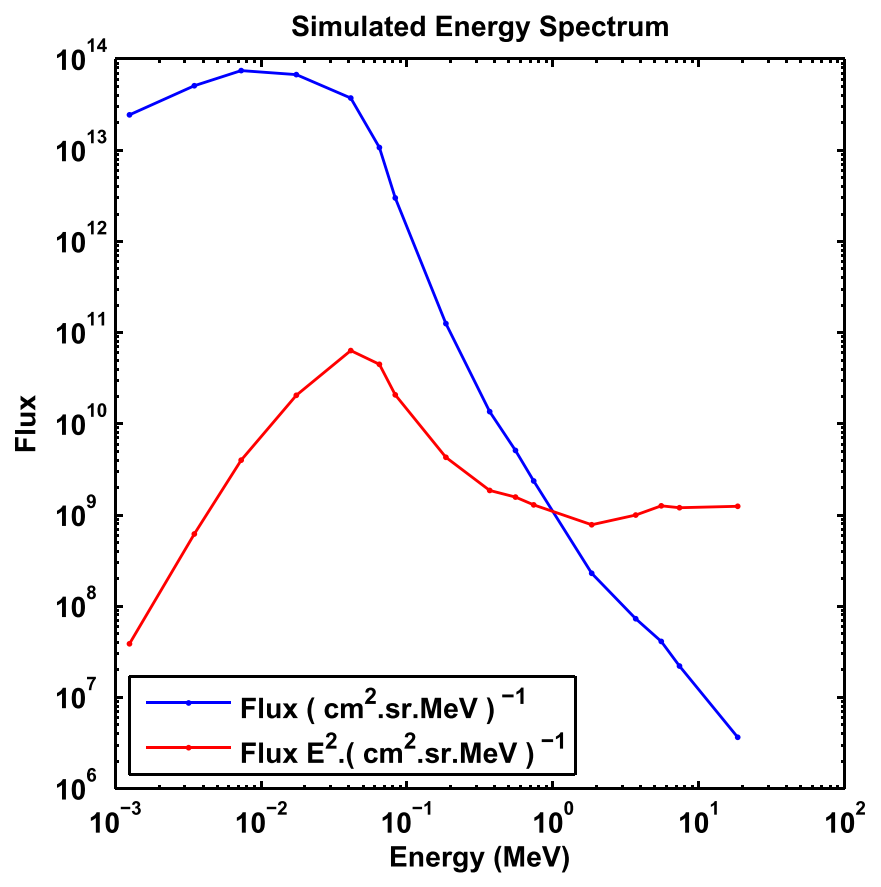

Figure 5. Simulated energy spectrum calculated from the twin-shock scenario. The blue curve represents the particle fluxes with the normal representation, and the red curve represents the energy spectrum by using a factor of the square of the energy times the fluxes.

energy $E^{2}$ as the representation with the energy $E$. Although the energy spectrum only covers the narrow energy range up to $20 \mathrm{MeV}$, the energy spectral shape exhibits very different behaviors in the specific energy ranges. There seemingly exists a Maxwellian thermal peak with a value of $\sim 8 \mathrm{keV}$. Then, the suprathermal tail is shown at the energy range from $\sim 70$ to $\sim 300 \mathrm{keV}$ with a very soft index of $\gamma_{1}=-3.4 \pm 0.17$. During the first half of the simulation time, initial thermal particles in the upstream bulk flow of shock No. 1 enter into the downstream region for diffusive processse and lead to the superthermal energy spectral tail. This process can be described as the kinetic energies of the upstream bulk flow translating into the thermal particles by heating and compressing, so the suprathermal tail forms as distributed by the power-law range from $\sim 70$ to $\sim 300 \mathrm{keV}$. This very soft index also would be contributed by the initial density compression rate $r_{\rho}$ between the upstream and downstream regions of the first shock. According to the simulated result of the compression rate in shock No. $1, r_{1}=1.17 \pm 0.01$, the index of the energy spectrum can be obtained from the formula as $\gamma_{1}=r_{1} / 2$ $\left(r_{1}-1\right)$. For the next stage, when the suprathermal particles with the size of the mean free path $\lambda_{\text {mfp }}$ are large enough to inject from the downstream region back into the upstream region, the suprathermal particles can be accelerated into the higher energetic particles by the multiple shock crossings. So at the energy range from $\sim 300 \mathrm{keV}$ to $\sim 2 \mathrm{MeV}$, the energy spectrum becomes a new power-law slope with a slightly soft index of $\gamma_{2}=-2.69 \pm 0.11$. In this energy range, the powerlaw index is regularly dominated by the single-shock acceleration (shock No. 1). And going to the next higher energy range from $\sim 2$ to $\sim 5.5 \mathrm{MeV}$, the energy spectrum abruptly shows a very hard power-law slope with an index of $\gamma_{3}=-1.79 \pm 0.05$. This process can be explained by the fact that the posterior shock begins to enter into the simulation box, and the turbulent magnetic field at the interaction region between the two shocks is amplified abruptly by the energetic particles injecting from shock No. 1 to shock No. 2. This kind of MFA and the reacceleration for seed energetic particles can change the existing soft energy spectrum into the very hard energy spectrum immediately. On the next duration at the highest energy range from $\sim 5.5$ to $\sim 20 \mathrm{MeV}$, the energy spectrum shows the power-law slope with a not-so-hard index of $\gamma_{4}=-1.90 \pm 0.05$. After the completion of the merged common region of the twin shock, the merged twin shock runs like a large isolated CME-driven shock, so the energy spectrum shows the behavior of a power law with a slightly hard index gradually. With the twin shock propagating in the interplanetary space, the dissipation of the ambient media can slow down the twin-shock speed, leading to a softer and softer energy spectrum. Totally, the energy spectrum exhibits different behaviors at the specific episodes of the twin-shock scenario. Especially, the entire energy spectrum shows a concave property at the range from $\sim 300 \mathrm{kev}$ to $\sim 5.5 \mathrm{MeV}$. At the highest energy range beyond $\sim 5.5 \mathrm{MeV}$, the energy spectrum still exhibits a slightly hard spectral slope until the end of the simulation. In other words, the total energy spectral slope produced by the twin-shock scenario indicates a soft-hard-hard mode, which is consistent with the twin-flare scenario.

\section{Discussions}

In this simulation, we should notice that the solar wind speed is varying with the heliosphere distances rapidly. The bulk speed of the CMEs would change by $\sim 100 \mathrm{~km} \mathrm{~s}^{-1}$ at the location close to the Sun between 1 and $7.5 R_{\odot}$. We set the speed of the background solar wind as an average value of $\sim 400 \mathrm{~km} \mathrm{~s}^{-1}$ without spatial dependence at this simulation domain. Actually, the simulation mainly focuses on the shock formation and evolution but not the CME motions themselves. Although the speed of the CME can be as high as $1000-2000 \mathrm{~km} \mathrm{~s}^{-1}$, the actual shock driven by the CME just evolutes with a comparatively slow velocity $V_{s h} \sim 200 \mathrm{~km} \mathrm{~s}^{-1}$ in the local shock reference frame. This twin-shock system spends several hours to evolute for the target of the large SEP production, which purely originated from the initial Maxwellian distributed particles in the background solar wind. As a result, we have obtained the power-law energy spectrum covering the energy range up to $\sim 20 \mathrm{MeV}$. According to the observation of the $\gamma$-rays in the first several decades of minutes, there would exist more than $100 \mathrm{MeV}$ protons. But the simulated results have a little deficit from the observations. This energy deficit would be contributed by the following reasons: The first reason would be that the largest energy particles escape from the FEB from the simulation system. Second, we should take the larger initial thermal velocity $v_{L}$ than the present value, which is the same as the one at $1 \mathrm{au}$ distance. Considering the difference of the IMF strength between the heliocentric distance $1 R_{\odot}$ and 1 au, the larger thermal velocity $v_{L}$ would be chosen. Third, if the collision time is taken to be a constant, the particles' mean free path would be proportional to their local velocities in the local reference frame. We let the collision time $\tau$ be equal to $7.14 \mathrm{~s}$ in the Sun surface region, which is different from the surface of Earth at $1 \mathrm{au}$. In fact, the collision time would also be varying with the turbulent magnetic field, so if we see the average collision time as a constant, it would not substantially affect the largest energy particle population. An additional reason would be that the difference of the parameter of background solar 
wind speed is set to be a constant, which seemingly should depend on radial distances. If we can consider all of the impacts of these parameters, we would obtain a more extensive energy spectrum. Besides the above reasons, we simulate the twin shock just for the first several hours, but the twin-sock system is still propagating forward to the interplanetary space and keeps on accelerating the particles, so the energy spectrum has not become the final energy spectrum, which is different from the observations from Earth.

\section{Summaries and Conclusions}

In summary, we have aimed to investigate the relationship between the GLE events and the SEP events and answering the following question: what type of shock events can trigger GLE events? According to the related observations from the multiple spacecraft based on the 2012 May 17 GLE event, we perform a twin-shock simulation for examining the difference of the energy spectrum between the twin shock and the single shock. According to the detailed simulation, we find that some behaviors of the twin shock are very different from those of a single shock. At the first half of the total simulation time, there is only a single shock propagating in the simulation box. But during the last half of the total simulation time, there are two shocks propagating into the simulation box. Simultaneously, there exists a common interaction region between two shocks that allows the accelerated energetic particles to be injected from the preceding shock into the posterior shock. Then, the common interaction region is gradually compressed by the relative movement between the double shock fronts until shock No. 1 disappears completely. At the episode of the common region compressing, the existing accelerated particles can be reaccelerated by the posterior shock abruptly. This merging interaction can turn the soft energy spectrum into the hard energy spectrum efficiently. So we can see that the energy spectrum exhibits different behaviors during the different episodes in the twin-shock scenario. Particularly, we find an energy spectral concave shape with the variation from the soft slope to the very hard slope at the energy range from $\sim 300 \mathrm{keV}$ to $\sim 5.5 \mathrm{MeV}$. At the energy range beyond $5.5 \mathrm{MeV}$, the energy spectrum still maintains a slightly hard slope until the end of the simulation. We suggest that the concave structure of the energy spectrum is very different from the normal energy spectrum produced by a single-shock scenario. By analyzing the concave structure of the energy spectrum, we basically think that the soft energy spectrum slope should be contributed by the preceding single shock, and the very hard energy spectrum slope is produced by the pileup collision between the posterior shock and the preceding shock. With the disappearance of the common compressed region, the merged twin shock still propagates forward like a large single shock into the interplanetary. The slightly hard energy spectrum at the energy range beyond $5.5 \mathrm{MeV}$ should be attributed to the pileup effect of the merged twin shock. But with the dissipation of the ambient media in further trips, it is estimated that the twinshock speed would be slow down gradually and the energy spectral slope would become softer and softer at the energy range beyond about a few hundred $\mathrm{MeV}$.

In conclusion, the SEP events produced by the twin-shock scenario would be responsible for triggering the related GLE events. So we suggest that the manner of the twin-shock interaction at the interplanetary space would become the prominent candidate for forecasting space weather. Since the twin-shock interaction is associated with the twin-CME sympathetic eruptions at the solar surface directly, the studies of the consecutive solar eruptions also would become popular topics of solar physics. For example, like twin-flare scenario at the solar atmosphere can also lead two-step of the magnetic reconnection for producing the soft-hard-hard mode of the electron energy spectrum. Here, we discuss the pileup encountering shocks at the interplanetary space that would amplify the turbulent magnetic field at the merged region of the twin shock, which can promote the acceleration efficiency for leading a concave energy spectrum (i.e., the soft-hard-hard slope) on the related SEP event. To an extent, both twin-CME and twin-flare types of eruptions can produce the concave shape on the related SEP energy spectra. In addition, we also compare the twin-shock and converging-shock types of double-shock scenarios on the aspect of the energy spectral shape in their corresponding SEP events. We find that the twin-shock scenario produces a concave energy spectrum on the related SEP event, but the converging-shock scenario provides a broken energy spectrum on its responding SEP event. At this point, we suggest that the shock interaction could produce quite different energy spectral structures from the normal energy spectrum contributed by a single-shock scenario.

This work is supported by the Xinjiang Natural Science Foundation No. 2019D01A100, Xinjiang Heaven Lake Hundred-Talent Program, and NSFC (No. 11673056). The present work is also supported by the CAS Key Laboratory of Solar Activity, National Astronomical Observatories; the Key Laboratory of Modern Astronomy and Astrophysics (Nanjing University), Ministry of Education; and the China Scholarship Council (CSC). Simultaneously, authors thank the support from the Supercomputer Center of University of Arizona. In addition, the authors appreciate Prof. J. R. Jokipii and his group at the University of Arizona; Prof. Hongbo Hu at IHEP; Prof. Xueshang Feng at NSSC; Prof. Jun Lin at YNAO; Profs. Yuming Wang, Chenlong Sun, and Quanming Lu at USTC in Chinese Academy of Sciences; and Profs. Zhengming Sheng and Suming Wen at Shanghai Jiao Tong University for their many helpful discussions. Finally, we also appreciate the reviewer's great comments for improving this paper. The referee's helpful points, advice, language tips, and valuable discussions are all included in this revision as much as possible.

\section{ORCID iDs}

Xin Wang (iD https://orcid.org/0000-0003-0116-2573

Mingde Ding (iD https://orcid.org/0000-0002-4978-4972

Chuan Li iD https://orcid.org/0000-0001-7693-4908

\section{References}

Adriani, O., Barbarino, G., Bazilevskaya, G., et al. 2015, ApJ, 801, A3 Ajello, M., Albert, A., Allafort, A., et al. 2014, ApJ, 789, 20

Asvestari, E., Willamo, T., Gil, A., et al. 2017, AdSpR, 60, 781 Axford, W. I., Leer, E., \& Skadron, G. 1977, ICRC (Plovdiv), 11, 132

Bamert, K., Kallenbach, R., Ness, N. F., et al. 2004, ApJL, 601, L99

Battarbee, M., Guo, J., Dalla, S., et al. 2018, A\&A, 612, A116

Bell, A. R. 1978, MNRAS, 182, 147

Blandford, R. D., \& Ostriker, J. P. 1978, ApJL, 221, L29

Bougeret, J. L., Kaiser, M. L., Kellogg, P. J., et al. 1995, SSRv, 71, 231

Bykov, A. M., Ellison, D. C., Osipov, S. M., \& Vladimirov, A. E. 2014, ApJ, 789,137

Cane, H. V., McGuire, R. E., \& von Rosenvinge, T. T. 1986, ApJ, 301, 448

Caprioli, D., Blasi, P., \& Amato, E. 2010, APh, 33, 307

Chen, C., Wang, Y., Shen, C., et al. 2011, JGRA, 116, A12108

Ding, J. Y., Hu, Y. Q., \& Wang, J. X. 2006, SoPh, 235, 223 
Ding, L. G., Jiang, Y., Zhao, L., \& Li, G. 2013, ApJ, 763, 30

Ding, L. G., Li, G., Jiang, Y., et al. 2014, ApJL, 793, L35

Ellison, D. C., Giacalone, J., Burgess, D., \& Schwartz, S. J. 1993, JGR, 98 21058

Ellison, D. C., Möbius, E., \& Paschmann, G. 1990, ApJ, 352, 376

Ellsion, D. C., Warren, D. C., \& Bykov, A. M. 2013, ApJ, 776, 46

Ellsion, D. C., Warren, D. C., \& Bykov, A. M. 2016, MNRAS, 456, 3090

Forbush, S. E. 1946, PhRv, 70, 771

Gopalswamy, N., Xie, H., Akiyama, S., et al. 2013, ApJL, 765, L30

Gopalswamy, N., Xie, H., Yashiro, S., et al. 2012, SSRv, 171, 23

Gopalswamy, N., Yashiro, S., Michäk, G., et al. 2002, ApJL, 572, L103

Gordon, B. E., Lee, M. A., Mobius, E., \& Trattner, K. J. 1999, JGR, 104, 28263

Gou, T., Veronig, A. M., Dickson, E. C., Hernandez-Perez, A., \& Liu, R. 2017, ApJL, 845, L1

Hassler, D., Zeitlin, C., Wimmer-Schweingruber, R., et al. 2012, SSRv, 170,503

Heber, B., Dresing, N., Dröge, W., et al. 2013, ICRC (Rio de Janeiro), 33, 1380

Howard, R. A., Moses, J. D., Vourlidas, A., et al. 2008, SSRv, 136, 67

Kahler, S. W., Cliver, E. W., Cane, H. V., et al. 1987, ICRC (Moscow), 20, 121

Kahler, S. W., Hildner, E., \& Van Hollebeke, M. A. I. 1978, SoPh, 57, 429

Kahler, S. W., Sheeley, N. R., Jr., Howard, R. A., et al. 1984, JGR, 89, 9683

Kahler, S. W., \& Vourlidas, A. 2013, ApJ, 769, 143

Klein, Karl-Ludwig, \& Dalla, S. 2017, SSRv, 212, 1107

Knerr, J. M., Jokipii, J. R., \& Ellison, D. C. 1996, ApJ, 458, 641

Krymsky, G. F. 1977, DoSSR, 243, 1306

Kühl, P., Banjac, S., Dresing, N., et al. 2015, A\&A, 576, A120

Lario, D., Aran, A., Gómez-Herrero, R., et al. 2013, ApJ, 767, 41

Lawrence, D. J., Peplowski, P. N., Feldman, W. C., Schwadron, N. A., \& Spence, H. E. 2016, JGR, 121, 7398

Lee, M. 1983, JGR, 88, 6109

Lee, M. 2005, ApJS, 158, 38
Leroy, M. M., Winske, D., Goodrich, C. C., Wu, C. S., \& Papadopouloos, K. 1982, JGR, 87, 5081

Li, C., Firoz, K. A., Sun, L. P., \& Miroshnichenko, L. I. 2013, ApJ, 770, 34 Li, G., Moore, R., Mewaldt, R. A., Zhao, L., \& Labrador, A. W. 2012, SSRv, 171,141

Li, G., Zank, G., \& Rice, W. 2003, JGRA, 108, 1082

Li, G., Zank, G., \& Rice, W. 2005, JGR, 110, A06104

Li, G., \& Zank, G. P. 2005, ICRC (Mumbai), 29, 173

Li, G., Zank, G. P., Verkhoglyadova, O., et al. 2009, ApJ, 702, 998

Lin, R. P., Dennis, B. R., Hurford, G. J., et al. 2002, SoPh, 210, 3

Liu, C., Lee, J., Karlicky, M., et al. 2009, ApJ, 703, 757

Mason, G. M., Mazur, J. E., \& Dwyer, J. R. 1999, ApJ, 525, L133

Müler-Mellin, R., Kunow, H., Fleißner, V., et al. 1995, SoPh, 162, 483

Ng, C., Reames, D., \& Tylka, A. 2003, ApJ, 591, 461

Nitta, N. V., Liu, Y., DeRosa, M. L., \& Nightingale, R. W. 2012, SSRv, 171,61

Picozza, P., Galper, A., Castellini, G., et al. 2007, APh, 27, 296

Reames, D. V. 1995, RvGeo, 33, 585

Reames, D. V. 1999, SSRv, 90, 413

Rice, W. K. M., Zank, G. P., \& Li, G. 2003, JGR, 108, 1369

Shen, C., Li, G., Kong, X., et al. 2013, ApJ, 763, 114

Thernisien, A. F. R., Vourlidas, A., \& Howard, R. A. 2009, SoPh, 256, 111

Treumann, R. A. 2009, A\&ARv, 17, 409

Wang, X., Giacalone, J., Yan, Y., et al. 2017, ApJ, 842, 74

Wang, X., Wang, N., \& Yan, Y. 2013, ApJS, 209, 18

Wang, X., \& Yan, Y. 2011, A\&A, 530, A92

Wang, X., \& Yan, Y. 2012, RAA, 12, 1535

Wang, X., Yan, Y., Ding, M., et al. 2016, RAA, 16, 32

Zank, G., Rice, W., \& Wu, C. 2000, JGR, 105, 25079

Zeitlin, C., Hassler, D., Cucinotta, F., et al. 2013, Sci, 340, 1080

Zhukov, A. N., \& Veselovsky, I. S. 2007, ApJL, 664, 131 